19

\title{
Конфигурационный резонанс и скорость генерации поверхностных плазмон-поляритонов возбужденными полупроводниковыми квантовыми точками вблизи металлической поверхности*
}

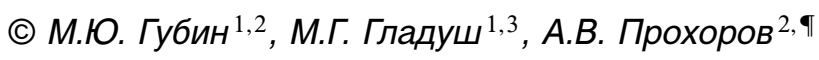 \\ ${ }^{1}$ Московский педагогический государственный университет, \\ 119991 Москва, Россия \\ ${ }^{2}$ Владимирский государственный университет им. А.Г. и Н.Г. Столетовых, \\ 600000 Владимир, Россия \\ ${ }^{3}$ Институт спектроскопии РАН, \\ 108840 Москва, Троицк, Россия \\ ฯ e-mail: avprokhorov33@mail.ru
}

Поступила в редакцию 24.09.2018 г.

Рассмотрены особенности генерации поверхностных плазмон-поляритонов в системе планарного интерфейса металл-диэлектрик, связанного ближнеполевым взаимодействием с внедренными в диэлектрик полупроводниковыми квантовыми точками. В качестве модели рабочей среды при проведении численного анализа использовалась золотая металлическая поверхность с нанесенной на нее диэлектрической пленкой (полиэтилентерефталат), содержащей сферическую квантовую точку на основе полупроводника CdSe. Решена задача оптимизации радиуса квантовой точки и ее расстояния до металлической поверхности для достижения максимальной эффективности передачи энергии квантовой точки для генерации поверхностных плазмон-поляритонов. Изучено влияние дисперсионных эффектов на скорость генерации поверхностных волн ансамблями неоднородных квантовых точек, расположенных в слое диэлектрика вблизи металлической поверхности.

DOI: $10.21883 /$ OS.2019.01.47059.254-18

\section{Введение}

Существенный вклад в теорию ближнеполевой микроскопии был сделан в работе [1], где была получена система самосогласованных уравнений для описания локального поля диэлектрического зонда, взаимодействующего с диэлектрической поверхностью. Авторами работы были определены условия для наблюдения конфигурационных резонансов, когда при определенных расстояниях до поверхности наблюдается усиление самосогласованного поля. В последующих работах в качестве зонда использовался металлический нанообъект, что расширяло возможности измерений, поскольку выбор размера частицы позволяет „управлять“ частотой плазмонного резонанса [2]. Однако спектральная ширина области, в которой наблюдается эффективная передача энергии, достаточна широка. Добиться ее сужения можно при использовании конфигурации типа одномодового квантового излучателя вблизи металлической поверхности. Наиболее эффективный обмен энергией в такой системе реализуется при условиях вблизи так называемого „плазмонного пика“, когда при заданном расстоянии от излучателя [3] до металлической поверхности происходит генерация поверхностных плазмон-поляри-

* XIII International Conference on Hole Burning, Single Molecule, and Related Spectroscopies: Science and Applications (HBSM-2018), August 6-12, 2018, Suzdal-Moscow, Russia. тонов (ППП). Однако использование атомов (молекул) в качестве таких квантовых излучателей автоматически означает отсутствие возможности управления частотой, на которой происходит эффективный энергообмен. Такая возможность возникает, если в качестве излучателей использовать полупроводниковые квантовые точки (КТ), размер которых определяет центральную частоту линии излучения. Такой подход определяет новые перспективы создания эффективных оптоплазмонных конвертеров и генераторов ППП.

В общем случае возбуждение ППП может осуществляться различными способами. Например, с использованием чисто геометрических схем типа схемы Кретчмана [4], в которой возбуждение ППП происходит при наклонном падении оптического луча на основание диэлектрической призмы с нанесенной на нее металлической пленкой. Такие схемы, в частности, лежат в основе сенсоров для измерения оптических свойств материалов $[5,6]$. Другие методы используют дифракцию света на брэгговских решетках для возбуждения ППП как в планарных $[7,8]$, так и в волоконно-оптических [9-12] системах. Однако в этих схемах низка эффективность конвертации энергии опорного оптического пучка в плазмонные моды.

Изучаемая в настоящей работе модель передачи энергии от возбужденной КТ к ППП основывается на подходах, изложенных в $[3,13,14]$. В этих работах 


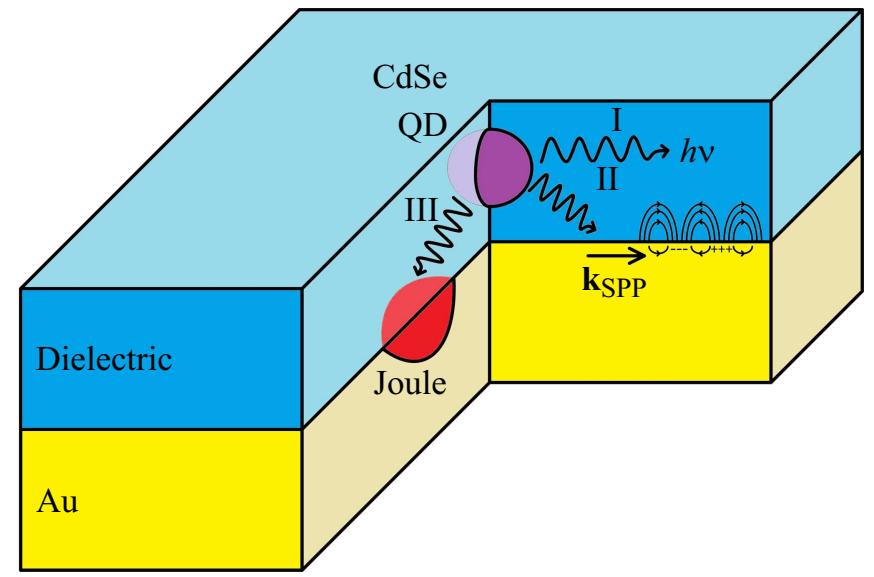

Рис. 1. Планарный интерфейс металл-диэлектрик с КТ для генерации ППП.

было показано, что релаксация возбужденной молекулы красителя вблизи металлической поверхности может осуществляться как через излучательные, так и безызлучательные каналы релаксации в зависимости от расстояния до нее. В частности, в работе [13] было найдено такое расстояние до металлической поверхности, что бо́льшая часть энергии излучателя ( 93\%) передавалась именно в плазмонные моды. Целью настоящей работы является анализ подобной системы, когда в качестве излучателей используются КТ. В такой постановке задачи существует возможность существенно расширить способы управления эффективностью, длиной волны и другими характеристиками генерируемых в системе ППП через подбор как размера самих КТ, так и их расстояния до металлической поверхности.

В практическом плане анализ описываемых конфигурационных резонансов необходим для оптимизации параметров генераторов ППП, не использующих в своей основе стандартные оптические схемы возбуждения. В частности, для генерации импульсных режимов ППП [15] могут быть задействованы коллективные когерентные эффекты в ансамбле КТ [16-18]. Такие схемы генерации поверхностных электромагнитных волн могут быть полезны и для формирования сложных плазмон-поляритонных локализованных структур $[19,20]$.

\section{Особенности развития релаксационных процессов для возбужденной КТ вблизи металлической поверхности}

На рис. 1 представлена модель планарного интерфейса, состоящего из толстого металлического основания и диэлектрической пленки с размещенной в ней полупроводниковой КТ. Полагаем, что КТ изначально переводится в возбужденное состояние, а кинетика последующего развития релаксационных процессов будет в основном определяться размерами КТ и ее расстоянием до металлической поверхности.

Для модели классического диполя эволюция дипольного момента $p$ межуровневого (межзонного) перехода КТ подчиняется классическому уравнению движения [3]:

$$
\ddot{p}+\omega_{0}^{2} p=\frac{e^{2}}{m} E_{R}-b_{0} \dot{p},
$$

где $e-$ заряд электрона, $\omega_{0}-$ собственная частота колебаний в отсутствие внешних воздействий, $m$ эффективная масса диполя, $E_{R}-$ напряженность поля, отраженного от металлического зеркала, $b_{0}$ - скорость затухания в отсутствие зеркала. Осуществляя переход от (1) к однородному дифференциальному уравнению (Приложение 1), получим следующие выражения для новых скоростей релаксации $b$ и частоты колебаний диполя $\omega$ :

$$
\begin{gathered}
b_{1}=\frac{b}{b_{0}}=1+\frac{3 q n_{1}^{2}}{2 p_{0} k_{1}^{3}} \operatorname{Im}\left(E_{0}\right), \\
\omega=\omega_{0}-\frac{e^{2} \operatorname{Re}\left(E_{0}\right)}{2 \omega_{0} m p_{0}}+\frac{b^{2}}{8 \omega_{0}}-\frac{b b_{0}}{4 \omega_{0}},
\end{gathered}
$$

где справедливы следующие обозначения:

$$
\begin{gathered}
p=p_{0} e^{(-b / 2-i \omega) t} \\
E_{R}=E_{0} e^{(-b / 2-i \omega) t}
\end{gathered}
$$

Здесь использовано соотношение $b_{r}=\frac{2 e^{2} k_{1}^{2}}{3 m \omega \omega_{1}^{2}}$, где $k_{1}=\omega n_{1} / c, n_{1}-$ показатель преломления содержащей диполь среды, $q=b_{r} / b-$ квантовый выход. Расчет напряженности поля $E_{0}$ основывается на нахождении вектора Герца при соблюдении условия непрерывности поля на границе раздела сред исходя из ориентации диполя относительно металлической поверхности [3]. Ограничимся рассмотрением ситуации, когда дипольный момент КТ ориентирован перпендикулярно поверхности зеркала. В этом случае выражение (2) принимает следующий вид [13]:

$$
\begin{aligned}
b_{\perp}= & +\frac{3}{2} q \operatorname{Im}\left[\int_{0}^{\infty} r_{p}\right. \\
& \left.\times \exp \left(\frac{-4 \pi \sqrt{\varepsilon_{1}} l_{1} d}{\lambda}\right) \frac{u^{3}}{l_{1}} \mathrm{~d} u\right],
\end{aligned}
$$

где введены коэффициенты $r_{p}=\frac{\varepsilon_{2} l_{1}-\varepsilon_{1} l_{2}}{\varepsilon_{2} l_{1}+\varepsilon_{1} l_{2}}$ (амплитудный коэффициент отражения $p$-поляризованного света Френеля от толстого слоя металла) и $l_{j}=-i \sqrt{\frac{\varepsilon_{j}}{\varepsilon_{1}}-u^{2}}$. 
Здесь $\varepsilon_{1}-$ диэлектрическая проницаемость диэлектрика, $\varepsilon_{2}=\varepsilon_{2}^{\prime}+i \varepsilon_{2}^{\prime \prime}-$ комплексная функция диэлектрической проницаемости металла на длине волны $\lambda_{0}$ флуоресцирующей КТ; переменная интегрирования $u=k / k_{0}$ представляет собой нормированный на $k_{0}=2 \pi / \lambda_{0}$ волновой вектор.

Особенность использования КТ в качестве излучателя заключается в необходимости учета размерных эффектов как для рабочей длины волны $\lambda_{0}$, так и дипольного момента $p_{0}$. Смещение значения $\lambda_{0}$ при изменении радиуса $a$ КТ влечет также изменение условий плазмонэкситонного резонанса. Поэтому возникает вопрос, насколько чувствительна скорость релаксации к дисперсии размера КТ и насколько точно необходимо выдерживать расстояние между КТ и металлическим зеркалом для достижения наилучших показателей.

Начнем с учета размерных эффектов, когда зависимость частоты межзонного перехода для КТ описывается выражением

$$
\omega_{n^{\prime} n}=\frac{1}{\hbar}\left(e E_{g}+\frac{\hbar^{2}}{2 a}\left(\frac{\chi_{n^{\prime} l^{\prime}}^{2}}{m_{e}}+\frac{\chi_{n l}^{2}}{m_{h}}\right)-\frac{3.56 e^{2}}{8 \pi \varepsilon \varepsilon_{0} a}\right),
$$

где $n^{\prime}(n)$ - главное, а $l^{\prime}(l)$ - орбитальное квантовые числа для электрона (дырки) с эффективными массами $m_{e}\left(m_{h}\right), \chi_{n l}-$ корни сферического уравнения Бесселя 1-го рода, $E_{g}$ - ширина запрещенной зоны полупроводника КТ, $\varepsilon$ - диэлектрическая проницаемость материала КТ.

Будем использовать полупроводниковые КТ малого радиуса, для которых наблюдаются сильные эффекты размерного квантования и появляются большие зазоры между соседними энергетическими уровнями как в валентной зоне, так и в зоне проводимости. Далее будем использовать только такие размеры КТ, при которых лишь один переход $1 S(e) \rightarrow 1 S(h)$ попадает в область так называемого плазмонного пика $[3,13]$, вызывая тем самым генерацию ППП вблизи заданной частоты:

$$
\omega_{0}=\frac{1}{\hbar}\left(e E_{g}+\frac{\hbar^{2} \pi^{2}}{2 a}\left(\frac{\chi_{n^{\prime} l^{\prime}}^{2}}{m_{e}}+\frac{\chi_{n l}^{2}}{m_{h}}\right)-\frac{3.56 e^{2}}{8 \pi \varepsilon \varepsilon_{0} a}\right) .
$$

Дипольный момент соответствующего перехода также будет проявлять, пусть и не столь сильную, зависимость от размера КТ. Эта зависимость проявляется косвенно, так как частота $\omega_{0}$ входит в соотношение для дипольного момента:

$$
p_{0}^{2}=\frac{e^{2}}{6 m_{0} \omega_{0}}\left(\frac{m_{0}}{m_{e}}-1\right) \frac{\omega_{0}\left(\omega_{0}+\Delta_{0}\right)}{\omega_{0}+\frac{2 \Delta_{0}}{3}},
$$

где $m_{0}-$ масса электрона, $\Delta_{0}-$ спин-орбитальное расщепление.

Настройка на условие плазмон-экситонного резонанса в нашей работе также осуществляется посредством учета зависимости диэлектрической проницаемости металла от частоты возбуждающего поля $\omega_{0}$, а следовательно, и от радиуса $а$ КТ. Мы используем формулу Друде в виде

$$
\varepsilon_{2}=1-\frac{\omega_{p l}^{2}}{\omega_{0}\left(\omega_{0}+i \gamma_{c}\right)} \text {, }
$$

где $\gamma_{c}-$ столкновительная частота, а $\omega_{p l}=\sqrt{\frac{n e^{2}}{m_{p l} \varepsilon_{0}}}-$ плазменная частота металла с концентрацией $n$ свободного электронного газа и эффективной массой электронов $m_{p l}$.

Далее нас будет интересовать вопрос увеличения эффективности генерации ППП в металле путем оптимизации размера КТ и точности их локализации в слое диэлектрика над металлом.

\section{Генерация ППП в процессе распада возбужденных КT вблизи металлического зеркала}

В общем случае имеются три канала релаксации для возбужденной КТ вблизи металлического зеркала: радиационная релаксация с генерацией фотонов, тепловые потери с генерацией фононов в толще металла и, наконец, нерадиационная прямая передача энергии КТ на возбуждение ППП на границе металл/диэлектрик через ближнее поле. Ключевым параметром для инициализации одного из этих трех путей является соотношение $u$ между волновым вектором генерируемого возбуждения и волновым вектором фотона в вакууме для равных частот. Распределение энергии между этими каналами зависит от вида подынтегральной функции $F \equiv r_{p} \exp \left(\frac{-4 \pi \sqrt{\varepsilon_{1}} l_{1} d}{\lambda}\right) \frac{u^{3}}{l_{1}} \quad$ в (4) и в модели рис. 1 может управляться через варьирование параметров $a$ и $d$.

Для анализа подынтегральной функции $F$ сначала фиксируем радиус КТ, к примеру, $a=2 \mathrm{~nm}$. В качестве материала КТ выбираем CdSe, в качестве металла выбираем золото, в качестве диэлектрика выбираем полиэтилентерефталат (ПЭТФ). Графики зависимости $F$ от $u$ при различных расстояниях $d$ до металлической поверхности представлены на рис. 2, $a$. Хорошо виден плазмонный пик, который ограничен слева значением $u=1$ для особой точки функции $F$. Вблизи нее для $u=u_{s p}=\operatorname{Re}\left(k_{s p} / k_{0}\right)$ наблюдается формирование волн ППП с волновым вектором $k_{s p}=k_{0} \sqrt{\frac{\varepsilon_{2}^{\prime} \varepsilon_{1}}{\varepsilon_{2}^{\prime}+\varepsilon_{1}}}$. Наличие дисперсии размывает область формирования ППП, правую границу которой можно оценить из простых соображений симметрии области генерации ППП вокруг $u_{s p}$, т. е. $u_{r}=u_{s p}+\left(u_{s p}-1\right)$ (рис. $\left.2, a\right)$. Вместе с тем зависимость $F$ от $u$ при разных значениях $a$ демонстрирует существенное сужение области плазмонного пика при использовании КТ меньших размеров.

Оценим вклад процессов генерации ППП через отношение площади плазмонного пика к общей площади под 

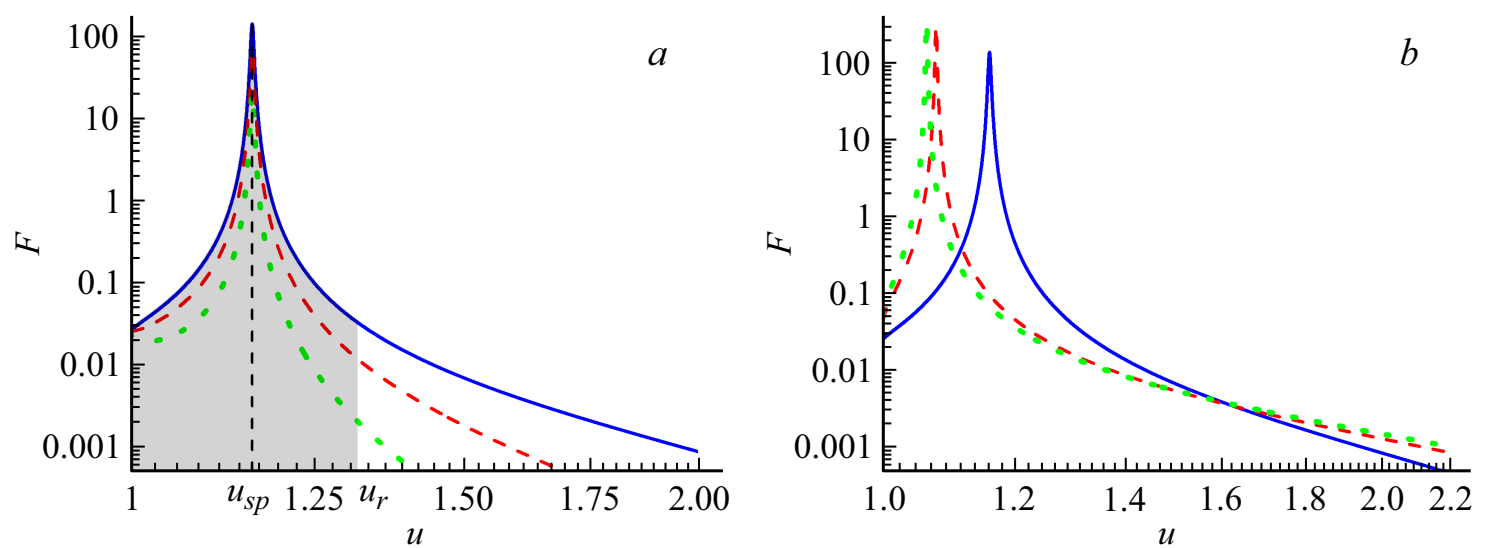

Рис. 2. Зависимости подынтегральной функции $F$ от отношения $u$ волнового вектора $k$ переизлученного фотона к волновому вектору $k_{0}$ фотона, возбуждающего КТ CdSe, $(a)$ при различных расстояниях $d$ от КТ до золотой поверхности: $d=d_{\text {орt }}=69$ (синяя сплошная кривая), 100 (красная штриховая кривая), $150 \mathrm{~nm}$ (зеленая пунктирная кривая), радиус КТ $a \equiv a_{1}=2 \mathrm{~nm}$; (b) при различных радиусах КТ: $a \equiv a_{1}=2$ (синяя сплошная кривая), $a \equiv a_{2}=4$ (красная штриховая кривая), $a \equiv a_{3}=10 \mathrm{~nm}$

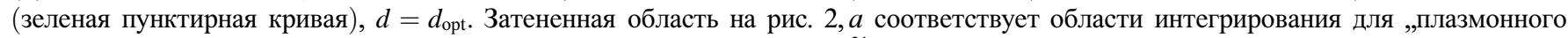
пика“. Параметры системы: $m_{e}=0.13 m_{0}, m_{h}=0.45 m_{0}, m_{0}=9.1 \cdot 10^{-31} \mathrm{~kg}$ - масса электрона, $E_{g}=1.7 \mathrm{eV}, \varepsilon=10, \Delta_{0}=0.62 \mathrm{eV}$, $\varepsilon_{1}=2.48, q=1, m_{p l}=1.224 m_{0}, n=5.91 \cdot 10^{28} \mathrm{~m}^{-3}, \gamma_{c}=4.1 \cdot 10^{13} \mathrm{~s}^{-1}$.

графиком на рис. 2, а. Для этого введем параметр интегральной вероятности передачи запасенной КТ энергии в ППП в виде $w_{p}=\frac{b_{s p}}{b_{\perp}}$, где

$$
\begin{aligned}
b_{s p}=1 & +\frac{3}{2} q \\
& \times \operatorname{Im}\left[\int_{1}^{2 u_{s p}-1} r_{p} \exp \left(\frac{-4 \pi \sqrt{\varepsilon_{1}} l_{1} d}{\lambda}\right) \frac{u^{3}}{l_{1}} \mathrm{~d} u\right] .
\end{aligned}
$$

В условиях $\varepsilon_{2}^{\prime \prime} \ll-\varepsilon_{2}^{\prime}$ соотношение (6) принимает следующий вид [13]:

$$
b_{s p}=\frac{3 q A \pi u_{s p}^{3}}{2 B l_{s p 1}} \exp \left(\frac{-4 \pi \sqrt{\varepsilon_{1}} l_{s p 1} d}{\lambda}\right),
$$

где

$$
\begin{gathered}
A=\frac{\left(2 l_{s p 2} \varepsilon_{1}-\frac{\varepsilon_{2}^{\prime}}{l_{s p 2}}\right) l_{s p 1}^{3} l_{s p 2}^{2} \varepsilon_{2}^{\prime \prime}}{\left(\varepsilon_{1} l_{s p 1}+\varepsilon_{2}^{\prime} l_{s p 2}\right)^{2} u_{s p}^{2}}, \\
B=\frac{\left(l_{s p 1}^{2} l_{s p 2}-\frac{1}{2} l_{s p 1}\right) \varepsilon_{2}^{\prime \prime}}{\left(\varepsilon_{1} l_{s p 1}+\varepsilon_{2}^{\prime} l_{s p 2}\right) u_{s p}}, \\
l_{s p i}=\sqrt{u_{s p}^{2}-\frac{\operatorname{Re}\left(\varepsilon_{i}\right)}{\varepsilon_{1}}}, \quad i=1,2 .
\end{gathered}
$$

На рис. 3 представлена контурная зависимость параметра $w_{p}$ от радиуса КТ и ее расстояния до металла. Нанесенная на рисунок черная линия соответствует максимальным значениям $w_{p}$, найденным по параметpy $d$. Наибольшие значения $w_{p} \approx 0.997$ достигаются

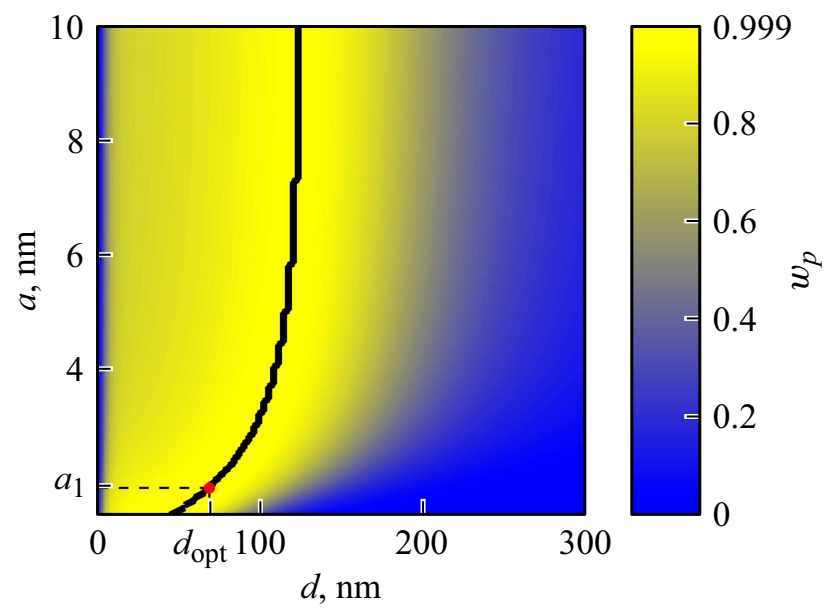

Рис. 3. Контур-плот для параметра эффективности $w_{p}$ преобразования энергии КТ в ППП в зависимости от радиуса КТ $a$ и расстояния КТ до металлического зеркала с нанесенной линией условного экстремума по параметру $d$. Расчет параметра $b_{\perp}$ производился численно с использованием метода замены переменных (Приложение 2). Параметры моделирования соответствуют рис. 2 .

для $a \approx 2 \mathrm{~nm}$ CdSe KT, размещенных на расстоянии $d \approx 69 \mathrm{~nm}$ от золотой поверхности. При этом снижение скорости генерации ППП в (7) при удалении КТ от поверхности может быть частично компенсировано увеличением длины волны $\lambda_{0}$ межзонного перехода при использовании КТ большего радиуса (5). Однако возможности такой компенсации ограничены наличием горизонтальной асимптоты $\omega_{0}=e E_{g} / \hbar$ в $(5)$ и, начиная с $a \approx 5 \mathrm{~nm}$, система становится слабо чувствительной к размерным эффектам для КТ, а положение максиму- 
ма $w_{p}$ стабилизируется вблизи $d \approx 120 \mathrm{~nm}$ для таких крупных (с $a>5 \mathrm{~nm}$ ) КТ. Таким образом, даже существенное увеличение радиуса для них (при $a>5 \mathrm{~nm}$ ) слабо повлияет на значения $w_{p}$. Далее будем полагать наличие ансамбля невзаимодействующих друг с другом КТ с различающимися радиусами, расположенных в слое диэлектрика заданной толщины над металлической поверхностью. Это необходимо для оценки изменения скорости генерации ППП в (неидеальных) условиях реального эксперимента.

Экспериментальная методика должна начинаться с подбора оптимального расстояния $d_{\text {орt }}$ для КТ с минимально возможным радиусом $a_{1}$ в ансамбле (рис. 4,a). Это связано с тем, что левое крыло функции эффективности генерации ППП имеет существенно более крутой спад для КТ малого размера. Оценим компактность размещения КТ в слое вблизи плоскости $d=d_{\text {opt }}$. К примеру, при значениях $a_{1}=2 \mathrm{~nm}$ выберем гауссово распределение КТ относительно плоскости $d_{\mathrm{opt}}=69 \mathrm{~nm}$ в виде

$$
\begin{aligned}
u_{d}(d)= & N\left(\sqrt { \frac { \pi } { 2 } } \sigma _ { d } \left(\operatorname{Erf}\left(\frac{d_{\mathrm{opt}}-d_{\mathrm{min}}}{\sqrt{2} \sigma_{d}}\right)\right.\right. \\
& \left.\left.-\operatorname{Erf}\left(\frac{d_{\mathrm{opt}}-d_{\mathrm{max}}}{\sqrt{2} \sigma_{d}}\right)\right)\right)^{-1} \exp \left(-\frac{\left(d-d_{\mathrm{opt}}\right)^{2}}{2 \sigma_{d}}\right),
\end{aligned}
$$

где $\operatorname{Erf}(x)=\frac{2}{\sqrt{\pi}} \int_{0}^{x} e^{-t^{2}} d t-$ функция Лапласа или функция ошибок, $N$ - количество КТ. Тогда наличие относительной дисперсии $\varepsilon_{d}=\sigma_{d} / d_{\mathrm{opt}}=20 \%$ для расстояний КТ до металлического зеркала (рис. 4,a) приведет к снижению эффективности генерации ППП на

$$
x_{d}=\left(1-\frac{\int_{0}^{\infty} w_{p}\left(a_{1}, d\right) u_{d}(d) \mathrm{d} d}{w_{p}\left(a_{1}, d_{\mathrm{opt}}\right)}\right) 100 \%=1.8 \% .
$$

Здесь $d_{\min }$ и $d_{\max }-$ минимальное и максимальное расстояния, на которых могут находится КТ, соответственно $d_{\min }=0 \mathrm{~nm}, d_{\max }=300 \mathrm{~nm}$.

На рис. 4, $b$ представлена зависимость параметра $w_{p}\left(a, d_{\text {opt }}\right)$ от размера КТ при фиксированном значении оптимизированного расстояния $d_{\text {орt }}$. По аналогии с pис. 4, a, выбирая относительную дисперсию радиуса КТ $\varepsilon_{a}=\sigma_{a} / a_{2}=20 \%$, получим снижение эффективности генерации ППП на

$$
x_{a}=\left(1-\frac{\int_{0}^{\infty} w_{p}\left(a, d_{\mathrm{opt}}\right) u_{a}(a) d a}{w_{p}\left(a_{1}, d_{\mathrm{opt}}\right)}\right) 100 \%=7.8 \% .
$$

Здесь

$$
\begin{aligned}
u_{a}(a)= & N\left(\sqrt { \frac { \pi } { 2 } } \sigma _ { a } \left(\operatorname{Erf}\left(\frac{a_{2}-a_{\min }}{\sqrt{2} \sigma_{a}}\right)\right.\right. \\
& \left.\left.-\operatorname{Erf}\left(\frac{a_{2}-a_{\max }}{\sqrt{2} \sigma_{a}}\right)\right)\right)^{-1} \exp \left(-\frac{\left(a-a_{2}\right)^{2}}{2 \sigma_{a}^{2}}\right)
\end{aligned}
$$

представляет гауссово распределение, где $a_{2}$ - среднее значение радиуса КТ, $a_{\min }$ и $a_{\max }-$ минимальное и максимальное значения радиуса КТ в ансамбле КТ, соответственно $a_{2}=4 \mathrm{~nm}, a_{\min }=1.5 \mathrm{~nm}, a_{\max }=10 \mathrm{~nm}$. Таким образом, разработка и создание интерфейсов для генерации ППП с рис. 1 должны учитывать сочетание относительно строгих требований к однородности КТ по размеру и более мягких требований по их размещению в слое диэлектрика.

Еще одним важным критерием при разработке подобных схем служит учет длины распространения ППП в интерфейсе:

$$
L_{S P P}=\frac{\lambda_{S P P}}{4 \pi} \frac{4 \varepsilon_{2}^{\prime}\left(\varepsilon_{2}^{\prime}+\varepsilon_{1}\right)}{\varepsilon_{2}^{\prime \prime} \varepsilon_{1}},
$$

где длина волны формируемых ППП

$$
\lambda_{S P P}=\lambda_{0} \sqrt{\frac{\operatorname{Re}\left(\varepsilon_{2}\right)+\varepsilon_{1}}{\operatorname{Re}\left(\varepsilon_{2}\right) \varepsilon_{1}}},
$$

$\lambda_{0}$ - длина волны электромагнитного излучения в вакууме. При этом волновой вектор для ППП равен $K_{S P P}=2 \pi / \lambda_{S P P}$. При точной настройке размещения КТ в диэлектрике на значении $d_{\text {opt }}$ дисперсионная и абсорбционная зависимости для комплексной диэлектрической проницаемости металла $\varepsilon_{2}$ на резонансной частоте ближнеполевого взаимодействия от радиуса КТ примут вид как на рис. 5, $a$. В совокупности с плавным ростом длины волны $\lambda_{S P P}$ для ППП на рис. 5, $b$ наблюдается монотонный рост параметра $L_{S P P}$ по мере увеличения $a$. В частности, при значении радиуса КТ $a_{2}$ длина пробега ППП составит $51 \mu \mathrm{m}$. В таблице представлены параметры системы и генерируемых ППП при различных значениях радиуса КТ. Следует отметить, что результаты приведенного моделирования можно считать лишь приближенными, так как более точная модель требует учета всей совокупности как меж-, так и внутризонных разрешенных переходов возбужденной КТ, а также релаксационных процессов между ними.

\section{Заключение}

Рассмотрена задача управления конфигурационными резонансами для полупроводниковой КТ, связанной ближнеполевым взаимодействием с металлической поверхностью. Выявлено наличие экстремумов скорости генерации ППП, связанных как с размером сферической КТ, так и ее расстоянием до металлической поверхности. Решена задача оптимизации скорости генерации ППП для CdSe KT вблизи золотой поверхности. С учетом успехов в области создания и сверхточной диагностики КТ [21], а также проектирования плазмоннных интерфейсов [22] полученные результаты должны быть востребованы для практического создания высокоэффективных генераторов ППП. 

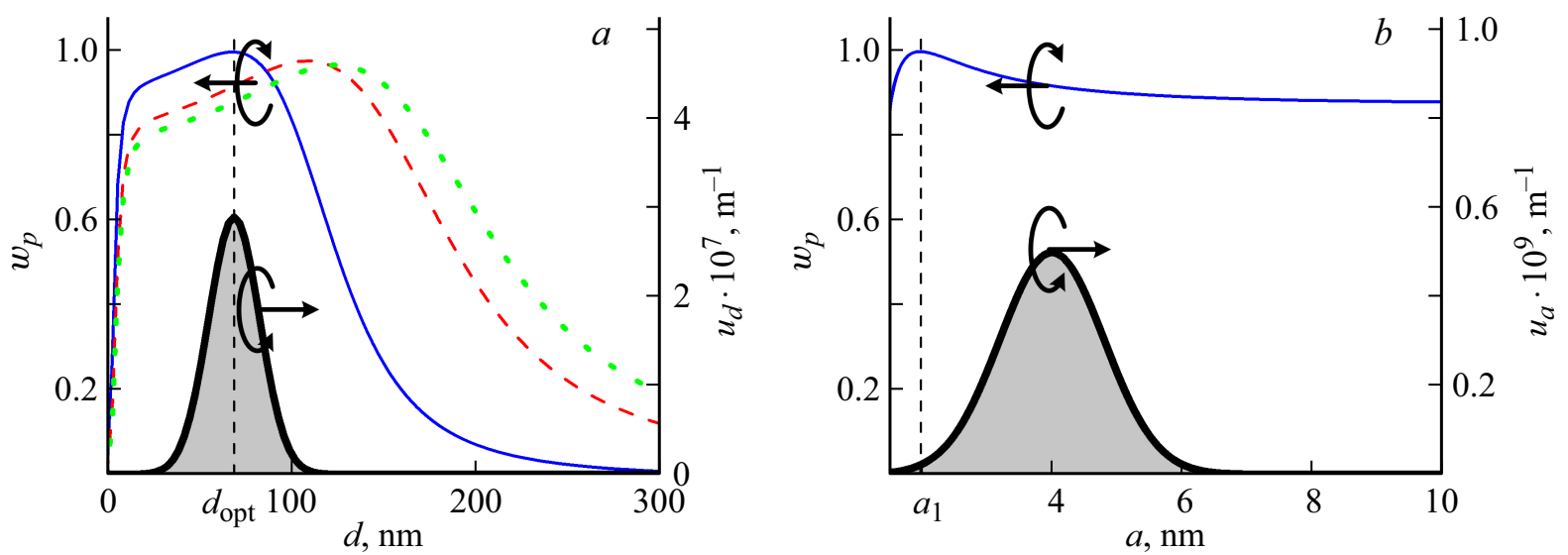

Рис. 4. Зависимости эффективности $w_{p}$ преобразования энергии КТ в ППП $(a)$ от расстояния до металлического зеркала для КТ с радиусами $a \equiv a_{1}=2$ (синяя сплошная кривая), $a \equiv a_{2}=4$ (красная штриховая кривая), $a \equiv a_{3}=10 \mathrm{~nm}$ (зеленая пунктирная

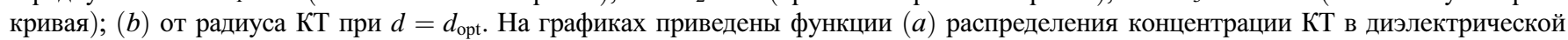
пленке над металлическим зеркалом, $(b)$ дисперсии КТ по радиусу. Параметры моделирования те же, что на рис. 2.
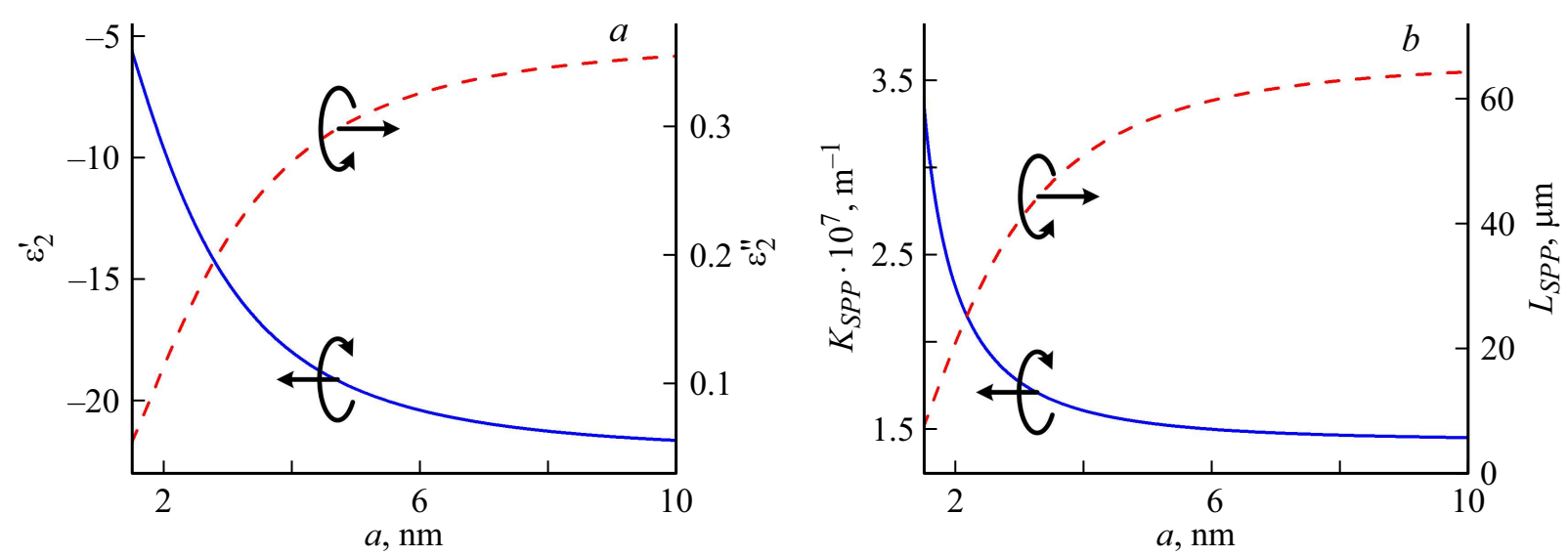

Рис. 5. Зависимости $(a)$ действительной и мнимой частей диэлектрической проницаемости металла, $(b)$ волнового вектора и длины затухания ППП от радиуса КТ, выступающих в качестве источника переизлученных электромагнитных волн. Параметры моделирования те же, что на рис. 2.

Параметры планарного интерфейса металл-диэлектрик с КТ CdSe и генерируемых ППП, параметры моделирования соответствуют рис. 2

\begin{tabular}{c|c|c|c|c|c|c|c}
\hline$a, \mathrm{~nm}$ & $d_{\mathrm{opt}}, \mathrm{nm}$ & $w_{p}$ & $\lambda, \mathrm{nm}$ & $L_{S P P}, \mu \mathrm{m}$ & $K_{S P P} \cdot 10^{7}, \mathrm{~m}^{-1}$ & $\varepsilon_{2}$ & $p_{0} \cdot 10^{-29}, \mathrm{~cm}$ \\
\hline 2 & 69 & 0.9969 & 497 & 21.0 & 2.31 & $-9.6634+0.1152 i$ & 3.05 \\
3 & 96 & 0.9840 & 610 & 40.3 & 1.78 & $-15.0472+0.2128 i$ & 3.40 \\
4 & 108 & 0.9760 & 663 & 51.0 & 1.61 & $-17.9637+0.2733 i$ & 3.55 \\
5 & 114 & 0.9717 & 688 & 56.5 & 1.54 & $-19.4486+0.3061 i$ & 3.63 \\
6 & 120 & 0.9691 & 703 & 59.7 & 1.50 & $-20.3318+0.3261 i$ & 3.67 \\
7 & 120 & 0.9677 & 711 & 61.6 & 1.48 & $-20.8445+0.3379 i$ & 3.69 \\
8 & 123 & 0.9667 & 717 & 62.9 & 1.47 & $-21.1865+0.3459 i$ & 3.71 \\
9 & 123 & 0.9661 & 720 & 63.7 & 1.46 & $-21.4047+0.3510 i$ & 3.72 \\
10 & 123 & 0.9656 & 723 & 64.3 & 1.46 & $-21.5616+0.3547 i$ & 3.72
\end{tabular}


Также выполнена оценка снижения эффективности генерации ППП при отклонении размеров КТ и их расстояния до металла от заданных значений. Установлено, что для таких неидеальных условий наибольшее влияние оказывает разброс радиуса КТ при относительно малом снижении скорости генерации ППП при отклонении расстояния КТ до металла от оптимальных значений. Развитие настоящей работы связано с решением задачи оптимизации генерации ППП для реальных устройств с учетом эффектов оптической диффузии в КТ [23], влияния коллективных эффектов локального поля [24,25], а также учета особенностей диполь-дипольных взаимодействий между отдельными хромофорами [26,27].

А.В. Прохоров благодарен профессору А.Б. Евлюхину за полезные обсуждения.

Работа поддержана грантом РФФИ 16-02-01174 а и выполнена в рамках государственного задания ВлГУ 3.5531.2017/8.9 (ГБ-1106/17).

\section{Приложение 1. \\ Вывод скоростей релаксации $b$ и частоты колебаний диполя $\omega$}

Сведем уравнение (1) к однородному дифференциальному уравнению и получим соотношения для новых скоростей релаксации и частотного сдвига. Подставляя решения (3) в уравнение (1), получим

$$
\begin{aligned}
& p_{0} e^{(-b / 2-i \omega) t}\left(-\frac{b}{2}-i \omega\right)^{2} \\
& +\omega_{0}^{2} p_{0} e^{(-b / 2-i \omega) t}=\frac{e^{2}}{m} E_{0} e^{(-b / 2-i \omega) t} \\
& -b_{0} p_{0} e^{(-b / 2-i \omega) t}\left(-\frac{b}{2}-i \omega\right) .
\end{aligned}
$$

Разделим это выражение на $e^{(-b / 2-i \omega) t}$, комплексную амплитуду $E_{0}$ представим в алгебраической форме $E_{0}=\operatorname{Re}\left(E_{0}\right)+i \operatorname{Im}\left(E_{0}\right)$ и получим

$$
\begin{aligned}
& p_{0}\left(-\frac{b}{2}-i \omega\right)^{2}+\omega_{0}^{2} p_{0} \\
& \quad=\frac{e^{2}}{m}\left(\operatorname{Re}\left(E_{0}\right)+i \operatorname{Im}\left(E_{0}\right)\right)-b_{0} p_{0}\left(-\frac{b}{2}-i \omega\right) .
\end{aligned}
$$

Разделим на мнимую и действительную части полученное выражение

$$
\begin{array}{r}
-\frac{e^{2} \operatorname{Re}\left(E_{0}\right)}{m}+\frac{p_{0} b^{2}}{4}-\frac{b b_{0} p_{0}}{2}-p_{0} \omega^{2}+\omega_{0}^{2} p_{0} \\
-i\left(-\frac{e^{2} \operatorname{Im}\left(E_{0}\right)}{m}+b p_{0} \omega-b_{0} p_{0} \omega\right)=0
\end{array}
$$

и получим систему уравнений

$$
\begin{gathered}
-i\left(-\frac{e^{2} \operatorname{Im}\left(E_{0}\right)}{m}+b p_{0} \omega-b_{0} p_{0} \omega\right)=0, \\
-\frac{e^{2} \operatorname{Re}\left(E_{0}\right)}{m}+\frac{p_{0} b^{2}}{4}-\frac{b b_{0} p_{0}}{2}-p_{0} \omega^{2}+\omega_{0}^{2} p_{0}=0 .
\end{gathered}
$$

Решая ее совместно, получим

$$
b=b_{0}+b_{0} \frac{e^{2} \operatorname{Im}\left(E_{0}\right)}{m p_{0} \omega b_{0}}
$$

$$
\Delta \omega \approx-\frac{e^{2} \operatorname{Re}\left(E_{0}\right)}{2 \omega_{0} m p_{0}}+\frac{b^{2}}{8 \omega_{0}}-\frac{b b_{0}}{4 \omega_{0}},
$$

полагая, что $\omega^{2}-\omega_{0}^{2}=\left(\omega-\omega_{0}\right)\left(\omega+\omega_{0}\right) \approx 2 \omega_{0} \Delta \omega$, где $\Delta \omega=\omega-\omega_{0}$. Тогда для частоты колебаний диполя $\omega$ получим следующее выражение:

$$
\omega \approx \omega_{0}-\frac{e^{2} \operatorname{Re}\left(E_{0}\right)}{2 \omega_{0} m p_{0}}+\frac{b^{2}}{8 \omega_{0}}-\frac{b b_{0}}{4 \omega_{0}} .
$$

\section{Приложение 2. \\ Расчет интеграла для скорости генерации ППП методом замены переменных}

Разделим несобственный интеграл 2-го рода в (4) на сумму интегралов с учетом того, что подынтегральная функция терпит бесконечный разрыв в точке $u=1$ :

$$
\begin{aligned}
b_{\perp}= & +3 / 2 q \operatorname{Im}\left[\lim _{\delta_{1} \rightarrow-0} \int_{0}^{1+\delta_{1}} r_{p} \exp \left(-4 \pi \sqrt{\varepsilon_{1}} l_{1} d / \lambda\right) u^{3} / l_{1} \mathrm{~d} u\right] \\
& +3 / 2 q \operatorname{Im}\left[\lim _{\delta_{2} \rightarrow+0} \int_{1+\delta_{2}}^{\infty} r_{p} \exp \left(-4 \pi \sqrt{\varepsilon_{1}} l_{1} d / \lambda\right) u^{3} / l_{1} \mathrm{~d} u\right] .
\end{aligned}
$$

Внесем $и$ под знак дифференциала:

$$
\begin{aligned}
b_{\perp}=1 & +3 / 2 q \operatorname{Im}\left[\lim _{\delta_{1} \rightarrow-0} \int_{0}^{1+\delta_{1}} \frac{r_{p}}{4 l_{1}} \exp \left(-4 \pi \sqrt{\varepsilon_{1}} l_{1} d / \lambda\right) \mathrm{d} u^{4}\right] \\
& +3 / 2 q \operatorname{Im}\left[\lim _{\delta_{2} \rightarrow+0} \int_{1+\delta_{2}}^{\infty} \frac{r_{p}}{4 l_{1}} \exp \left(-4 \pi \sqrt{\varepsilon_{1}} l_{1} d / \lambda\right) \mathrm{d} u^{4}\right] .
\end{aligned}
$$


Сделаем замену переменной $u^{4}=x_{1}$ в (B1), получим

$$
\begin{aligned}
& b_{\perp}=1+3 / 2 q \operatorname{Im}\left[\lim _{\delta_{1} \rightarrow-0} \int_{0}^{1+\delta_{1}} \frac{r_{p}\left(x_{1}\right)}{4 l_{1}\left(x_{1}\right)}\right. \\
& \left.\times \exp \left(-4 \pi \sqrt{\varepsilon_{1}} l_{1}\left(x_{1}\right) d / \lambda\right) \mathrm{d} x_{1}\right] \\
& +3 / 2 q \operatorname{Im}\left[\lim _{\delta_{2} \rightarrow+0} \int_{1+\delta_{2}}^{\infty} \frac{r_{p}\left(x_{1}\right)}{4 l_{1}\left(x_{1}\right)}\right. \\
& \left.\times \exp \left(-4 \pi \sqrt{\varepsilon_{1}} l_{1}\left(x_{1}\right) d / \lambda\right) \mathrm{d} x_{1}\right]
\end{aligned}
$$

где

$$
\begin{gathered}
r_{p}\left(x_{1}\right)=\frac{\varepsilon_{2} l_{1}\left(x_{1}\right)-\varepsilon_{1} l_{2}\left(x_{1}\right)}{\varepsilon_{2} l_{1}\left(x_{1}\right)+\varepsilon_{1} l_{2}\left(x_{1}\right)}, \\
l_{1}\left(x_{1}\right)=-i \sqrt{1-\sqrt{x_{1}}}, \\
l_{2}\left(x_{1}\right)=-i \sqrt{\varepsilon_{2} / \varepsilon_{1}-\sqrt{x_{1}}} .
\end{gathered}
$$

Сделаем замены переменных в (В2) - во втором слагаемом $t_{1}=\sqrt{1-\sqrt{x_{1}}}\left(x_{1}=\left(1-t_{1}^{2}\right)^{2}\right)$ и в третьем слагаемом $t_{1}^{\prime}=\sqrt{\sqrt{x_{1}}-1}\left(x_{1}=\left(t_{1}^{\prime 2}+1\right)^{2}\right)$ :

$$
\begin{aligned}
& b_{\perp}=1+3 / 2 q \operatorname{Im}\left[\lim _{\delta_{1} \rightarrow-0} \int_{1}^{0-\delta_{1}}-i r_{p}\left(t_{1}\right)\right. \\
& \left.\times \exp \left(4 \pi \sqrt{\varepsilon_{1}} i t_{1} d / \lambda\right)\left(1-t_{1}^{2}\right) \mathrm{d} t_{1}\right] \\
& +3 / 2 q \operatorname{Im}\left[\lim _{\delta_{2} \rightarrow+0} \int_{0+\delta_{2}}^{\infty} r_{p}\left(t_{1}^{\prime}\right) \exp \left(-4 \pi \sqrt{\varepsilon_{1}} t_{1}^{\prime} d / \lambda\right)\left(t_{1}^{\prime 2}+1\right) \mathrm{d} t_{1}^{\prime}\right]
\end{aligned}
$$

где

$$
\begin{gathered}
r_{p}\left(t_{1}\right)=\frac{\varepsilon_{2} l_{1}\left(t_{1}\right)-\varepsilon_{1} l_{2}\left(t_{1}\right)}{\varepsilon_{2} l_{1}\left(t_{1}\right)+\varepsilon_{1} l_{2}\left(t_{1}\right)}, \\
l_{1}\left(t_{1}\right)=-i t_{1}, \\
l_{2}\left(t_{1}\right)=-i \sqrt{\varepsilon_{2} / \varepsilon_{1}-1+t_{1}^{2}}, \\
r_{p}\left(t_{1}^{\prime}\right)=\frac{\varepsilon_{2} l_{1}\left(t_{1}^{\prime}\right)-\varepsilon_{1} l_{2}\left(t_{1}^{\prime}\right)}{\varepsilon_{2} l_{1}\left(t_{1}^{\prime}\right)+\varepsilon_{1} l_{2}\left(t_{1}^{\prime}\right)}, \\
l_{1}\left(t_{1}^{\prime}\right)=t_{1}^{\prime}, \quad l_{2}\left(t_{1}^{\prime}\right)=-i \sqrt{\varepsilon_{2} / \varepsilon_{1}-t_{1}^{\prime 2}-1} .
\end{gathered}
$$

\section{Список литературы}

[1] Keller O., Xiao M., Bozhevolnyi S. // Surf. Sci. 1993. V. 280. N 1-2. P. 217. doi 10.1016/0039-6028(93)90370-Y

[2] Xiao M., Bozhevolnyi S., Keller O. // Appl. Phys. A. 1996. V. 62. N 2. P. 115. doi 10.1007/BF01575709
[3] Chance R.R., Prock A., Silbey R. Molecular fluorescence and energy transfer near interfaces. In: Prigogine I., Rice S.A., eds. Adv. Chem. Phys. V. 37. NY.: Wiley, 1978. 65 p. doi 10.1002/9780470142561.ch1

[4] Kretschmann E. // Z. Physik. 1971. V. 241. N 4. P. 313. doi 10.1007/BF01395428

[5] Raether H. Surface plasmons on smooth and rough surfaces and on gratings. Berlin: Springer-Verlag, 1988. 136 p. doi $10.1007 / \mathrm{BFb} 0048317$

[6] Kretschmann E. // Opt. Commun. 1972. V. 6. N 2. P. 185.

[7] Huang D., Easter M., Gumbs G., Maradudin A.A., Lin S.-Y., Cardimona D.A., Zhang X. // Opt. Express. 2014. V. 22. N 22. P. 27576. doi 10.1364/OE.22.027576

[8] Reinhardt C., Evlyukhin A.B., Cheng W., Birr T., Markov A., Ung B., Skorobogatiy M., Chichkov B.N. // JOSA. B. 2013. V. 30. N 11. P. 2898. doi 10.1364/JOSAB.30.002898

[9] Erdogan T. // JOSA. A. 1997. V. 14. N 8. P. 1760. doi 10.1364/JOSAA.14.001760

[10] Thomas J., Jovanovic N., Becker R.G., Marshall G.D., Withford M.J., Tünnermann A., Nolte S., Steel M. // Opt. Express. 2011. V. 19. N 1. P. 325. doi 10.1364/OE.19.000325

[11] Albert J., Shao L.Y., Caucheteur C. // Laser Photonics Rev. 2013. V. 7. N 1. P. 83. doi 10.1002/lpor.201100039

[12] Shevchenko Y.Y., Albert J. // Opt. Lett. 2007. V. 32. N 3. P. 211. doi 10.1364/OL.32.000211

[13] Weber W.H., Eagen C.F. // Opt. Lett. 1979. V. 4. N 8. P. 236. doi 10.1364/OL.4.000236

[14] Barnes W.L. // J. Mod. Opt. 1998. V. 45. N 4. P. 661. doi 10.1080/09500349808230614

[15] Khokhlov N.E., Ignatyeva D.O., Belotelov V.I. // Opt. Express. 2014. V. 22. N 23. P. 28019. doi 10.1364/OE.22.028019

[16] Babenko Ia.A., Yugova I.A., Poltavtsev S.V., Salewski M., Akimov I.A., Kamp M., Höfling S., Yakovlev D.R., Bayer M. // Semiconductors. 2018. V. 52. N 4. P. 531. doi $10.1134 / \mathrm{S} 106378261804005 \mathrm{X}$

[17] Аржсанов А.И., Каримуллин К.Р., Наумов А.В. // Краткие сообщения по физике ФИАН. 2018. Т. 45. № 3. C. 39. doi 10.3103/S1068335618030077; Arzhanov A.I., Karimullin K.R., Naumov A.V. // Bull. Lebedev Phys. Inst. 2018. V. 45. N 3. P. 91. doi 10.3103/S1068335618030077

[18] Шестериков А.В., Губин М.Ю., Гладуш М.Г., Прохоров А.В. // ЖЭТФ. 2017. Т. 151. № 1. С. 24. doi 10.7868/S0044451017010035; Shesterikov A.V., Gubin M.Yu., Karpov S.N., Prokhorov A.V. // JETP Lett. 2018. V. 107. N 7. P. 435. doi 10.1134/S0021364018070081

[19] Dzedolik I.V., Pereskokov V. // JOSA. A. 2018. V. 35. N 8. P. 1420. doi 10.1364/JOSAA.35.001420

[20] Dzedolik I.V. // J. Opt. 2014. V. 16. N 12. P. 125002. doi 10.1088/2040-8978/16/12/125002

[21] Magaryan K.A., Mikhailov M.A., Karimullin K.R., Knyazev M.V., Eremchev I.Y., Naumov A.V., Vasilieva I.A., Klimusheva G.V. // J. Lumin. 2016. V. 169. P. 799. doi 10.1016/j.jlumin.2015.08.064

[22] Birr T., Zywietz U., Fischer T., Chhantyal P., Evlyukhin A.B., Chichkov B.N., Reinhardt C. // Appl. Phys. B. 2016. V. 122. N 6. P. 164. doi 10.1007/s00340-016-6437-5

[23] Савостьянов А.О., Еремчев И.Ю., Горшелев А.А., Наумов А.В., Старухин А.С. // Письма в ЖЭТФ. 2018. Т. 107. № 7. C. 426. doi 10.7868/S0370274X18070044; Savostianov A.O., Eremchev I.Yu., Gorshelev A.A., Naumov A.V., Starukhin A.S. // JETP Lett. 2018. V. 107. N 7. P. 406. doi 10.1134/S002136401807007X 
[24] Губин М.Ю., Шестериков А.В., Гладуш М.Г., Прохоров А.В. // Известия РАН. Сер. Физ. 2017. Т. 81. № 12. C. 1704. doi 10.7868/S0367676517120274; Gubin M.Yu., Shesterikov A.V., Gladush M.G., Prokhorov A.V. // Bull. Russ. Acad. Sci. Phys. 2017. V. 81. N 12. P. 1507. doi 10.3103/S1062873817120139

[25] Kuraptsev A.S., Sokolov I.M. // Phys. Rev. A. 2014. V. 90. N 1. P. 012511. doi 10.1103/PhysRevA.90.012511

[26] Kuraptsev A.S., Sokolov I.M. // Laser Phys. 2018. V. 28. N 8. P. 085203. doi 10.1088/1555-6611/aac508

[27] Gubin M.Yu., Shesterikov A.V., Karpov S.N., Prokhorov A.V. /I Phys. Rev. B. 2018. V. 97. N 8. P. 085431.

doi 10.1103/PhysRevB.97.085431 